\title{
THE ULTRAVIOLET STARLIGHT IN THE GALAXY
}

\author{
P. M. Gondhalekar \\ Rutherford Appleton Laboratory \\ Chilton, Didcot, OXON \\ OX11 0QX UK
}

ABSTRACT. The distribution of UV bright stars in the Galaxy is described. The integrated ultraviolet (UV) stellar radiation in the Galaxy is obtained from these data, and this flux of radiation is compared with observations and models of direct starlight in the Galaxy.

\section{INTRODUCTION}

The sources of UV radiation in the Galaxy that have been discussed in the literature with various degrees of confidence are given in Table 1. These sources have been listed in order of decreasing emitted flux and increasing incredulity. The stellar population is the strongest source of UV radiation in the Galaxy, and this is discussed here. The other sources of UV radiation in the Galaxy have been discussed by various authors (e.g., Witt, Lequeux, Paresce, Bowyer, all 1989, this volume).

TABLE 1. Possible Sources of UV Radiation in the Galaxy

\begin{tabular}{l}
\hline \hline Direct star light \\
Starlight scattered off interstellar dust \\
Integrated radiation from extragalactic point sources \\
Emission from hot interstellar gas \\
Fluorescing molecular hydrogen \\
Redshifted line emission at earlier epochs \\
Radiative decay of -inos
\end{tabular}

It is now well established that stars of spectral type $\mathbf{O}$ to $\mathbf{G}$ are luminous in the UV (viz. the Lyman limit to $-3000 \AA$ ) and that the UV starlight in the Galaxy will be determined by the population and the distribution of these stars in the Galaxy. In the last twenty years, a number of attempts have been made to model or measure the flux of integrated UV starlight in the Galaxy (Table 2), but there seems to have been something of a lull in this activity in the last few years. Since the UV radiation is absorbed in the Earth's atmosphere, this radiation from the stars has to be observed from above the atmosphere. Also, to obtain a detailed distribution of radiation, it is necessary to make observations over the whole sky and to a sufficient depth. An all sky-survey is particularly important because the dominant emitters of UV radiation, particularly the hard $(\sim 1000 \AA)$ radiation, are early-type high-mass stars. The population of these stars is small, and they are widely distributed; therefore, there will be local enhancements of radiation. The second reason for an all sky-survey is the patchy nature of dust clouds in the Galaxy. The extinction due to dust is high in the UV, and starlight will be significantly attenuated even by small concentrations of dust in the line-of-sight. Because of this attenuation, the integrated radiation expected from stars in stellar catalogues can only be approximate 
as it would be practically impossible to build the details of dust distribution in the Galaxy into such a model. Also, there is no single extinction law in all directions in the Galaxy, and this adds a higher dimension of uncertainty in the galactic UV stellar radiation obtained from visible star catalogues.

TABLE 2. Observations and Models of Stellar UV Radiation in the Galaxy

\begin{tabular}{lll}
\hline \hline Habing & 1968 & model \\
Kurt and Sunyaev & 1968 & observations \\
Hayakawa, Yamashita, and Yoshioka & 1969 & observations \\
Belyaev et al. & 1971 & observations \\
Witt and Johnson & 1973 & model \\
Jura & 1974 & model \\
Grewing et al. & 1975 & model \\
Gondhalekar and Wilson & 1975 & model \\
Henry et al. & 1977 & observations \\
Henry & 1977 & BSC and SAO catalogues \\
Sandel, Shemansky, and Broadfoot & 1979 & observations \\
Gondhalekar, Phillips, and Wilson & 1980 & UV catalogue \\
Bixler, Bowyer, and Grewing & 1984 & observations \\
\hline
\end{tabular}

In the last twenty years, a number of rocket and satellite experiments have been launched to detect UV radiation emitted by stars. The rocket observations were only of a few stars (for obvious reasons), and most of the satellite experiments were operated in a "pointed mode" and observed rather a small number of selected stars. Only the $S 2 / 68$ experiment on board the $T D 1$ satellite and the $A N S$ and IUE satellites have observed stars in sufficient numbers to attempt to obtain a statistical average of direct UV stellar radiation in the Galaxy. The TD1-S2/68 observations are the best for this purpose as there was no observational bias in the data; also, this experiment has observed the largest number of stars in the UV. The stellar UV radiation in the Galaxy inferred from this survey is described here. The distribution of UV bright stars is described in Section 2. The distribution of stellar UV radiation in the Galaxy is described in Section 3, and the models and observations of the integrated starlight are discussed in Section 4. The conclusions are given in Section 5.

\section{THE ULTRAVIOLET OBSERVATIONS}

The European Space Research Organization (predecessor of the European Space Agency) satellite TDI was launched in March 1972. On board this satellite there was a UV astronomy experiment, called S2/68, which was provided by scientists from Belgium and the United Kingdom. The experiment has been described by Boksenberg et al. (1973). The TDI satellite was in a Sun-synchronous orbit, and the S2/68 experiment scanned the whole sky in six months; the spacecraft was operational for over two years. For a point source, the experiment provided broadband $(\Delta \lambda \approx 300)$ flux measurements at $2740 \AA$ and spectrophotometric data in three channels centered on $1565 \AA, 1965 \AA$, and $2365 \AA$; the width of each channel was $\sim 330 \AA$. To obtain the integrated starlight, the data in these spectrophotometric channels were combined, hence improving the statistical accuracy. The absolute calibration of the S2/68 experiment was based on rocket observations of $\eta$ UMa (Carnochan 1982), and the photometry is accurate to $\sim 10 \%$. 
A great body of stellar UV data from this experiment has been published in three catalogues (Jamar et al. 1976; Macau-Hercot et al. 1978; Thompson et al. 1978), and the distribution of stellar UV radiation over the whole sky has been discussed by Gondhalekar, Phillips, and Wilson (1980). However, the absolute calibration of the S2/68 experiment has since been revised, and it is now known that the detectors of this experiment were nonlinear for fluxes lower than $1.0 \times 10^{-12} \mathrm{ergs} \mathrm{cm}^{-2} \mathrm{~s}^{-1} \AA^{-1}$ (Carnochan 1989, private communication). Deeper maps of stellar UV radiation in limited regions of the sky have been obtained in the last few years (Page, Carruthers, and Heckathorn 1982; Carruthers and Page 1984; Onaka et al. 1989), but a detailed and accurate all sky-survey is not yet available.

\section{STAR DISTRIBUTION}

The complete (unpublished) TD1-S2/68 catalogue of 58,012 stars was used to obtain the global distribution of stellar UV radiation in the Galaxy. But only stars with flux greater than $1.0 \times 10^{-12}$ ergs cm $\mathrm{cm}^{-2} \mathrm{~s}^{-1} \AA^{-1}$ in one of the four S2/68 bands (given above) were selected for this study. This reduced the number of stars to 47,039 , and the distribution of these stars by galactic coordinates (as given in the catalogue) is shown in Figure 1. The well-defined semicircular patch centered on $l=300^{\circ}$ is most probably "instrumental," although there is no obvious cause for this artifact. The stars are concentrated along the galactic plane, as expected. A number of stellar associations can be seen in this distribution of stars; however, there is no gross asymmetry between the northern and southern hemispheres of the Galaxy, nor is there an east-west asymmetry in the distribution of stars.

The distribution of stars with the catalogued visual magnitude and the UV magnitudes (obtained from the observed fluxes) is shown in Figure 2. The UV fluxes were put on a visual magnitude scale using the calibration of Hayes and Lathem (1975) through the relation

$$
m(\lambda)=-2.5 \log F(\lambda)-21.175 \text {. }
$$

The turnover at $m_{\mathrm{v}} \approx 9$ in the distribution of visible magnitudes indicates that the survey is not complete for fainter stars. The cutoff in the UV magnitudes at $m_{\mathrm{uv}} \approx 9$ is due to the flux limit of $1.0 \times 10^{-12}$ ergs cm$~^{-2} \mathrm{~s}^{-1} \AA^{-1}$ imposed during selection of these stars. However, the turnover at $m_{\mathrm{uv}} \approx 8$ in the UV magnitudes suggests that the catalogue is complete only for stars brighter than $m_{\mathrm{uv}} \approx 8$ and that the flux limit is not likely to compromise the integrated flux of bright stars derived from these data. The distribution of visible and UV colors of these stars is given in Figure 3. Only stars of known $(B-V)$ and UV flux greater than $1.0 \times 10^{-12}$ ergs cm $\mathrm{cm}^{-2} \mathrm{~s}^{-1} \AA^{-1}$ in all four $\mathrm{S} 2 / 68$ bands were selected. The distribution of colors peaks around zero; this suggests that the integrated UV starlight of the Galaxy is similar to the light emitted by an A0 star. The colors also indicate that there is a large population of blue stars. The long red tail in these colour distributions also suggests a population of either reddened early-type stars or unreddened late-type stars in this catalogue.

\section{ULTRAVIOLET STELLAR RADIATION}

\subsection{Global Distribution}

The large number of detections of UV radiation emitted by stars can be used to obtain the global distribution of UV bright stars in the Galaxy. The intensity of stellar radiation averaged 


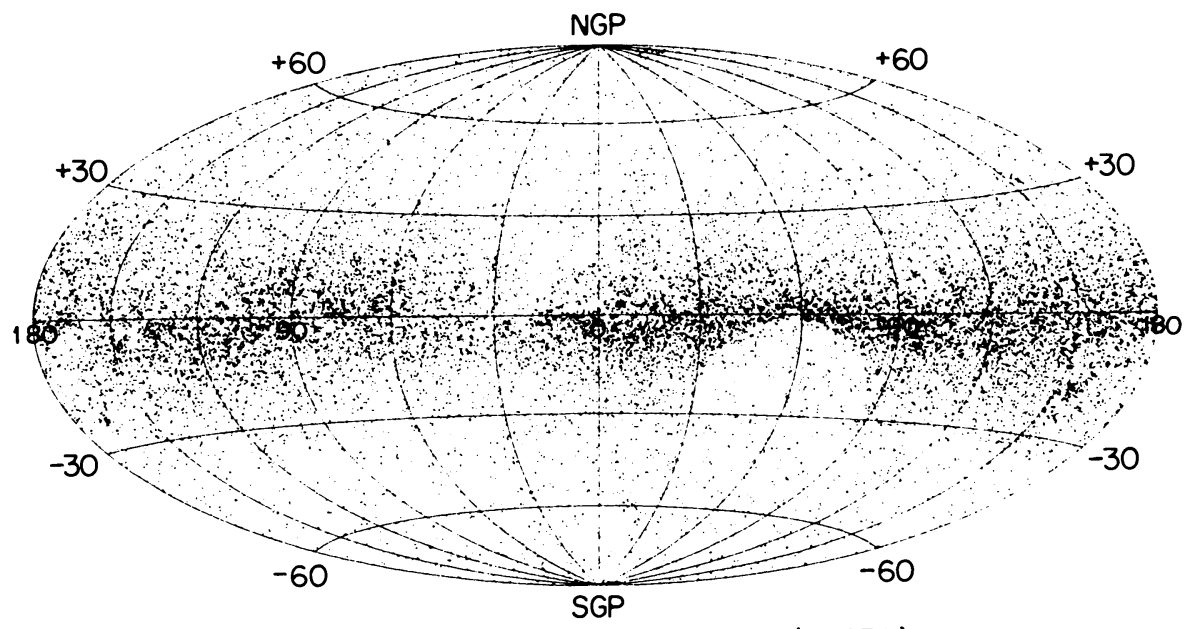

S268 CATALOGUE: ALL STARS (47039)

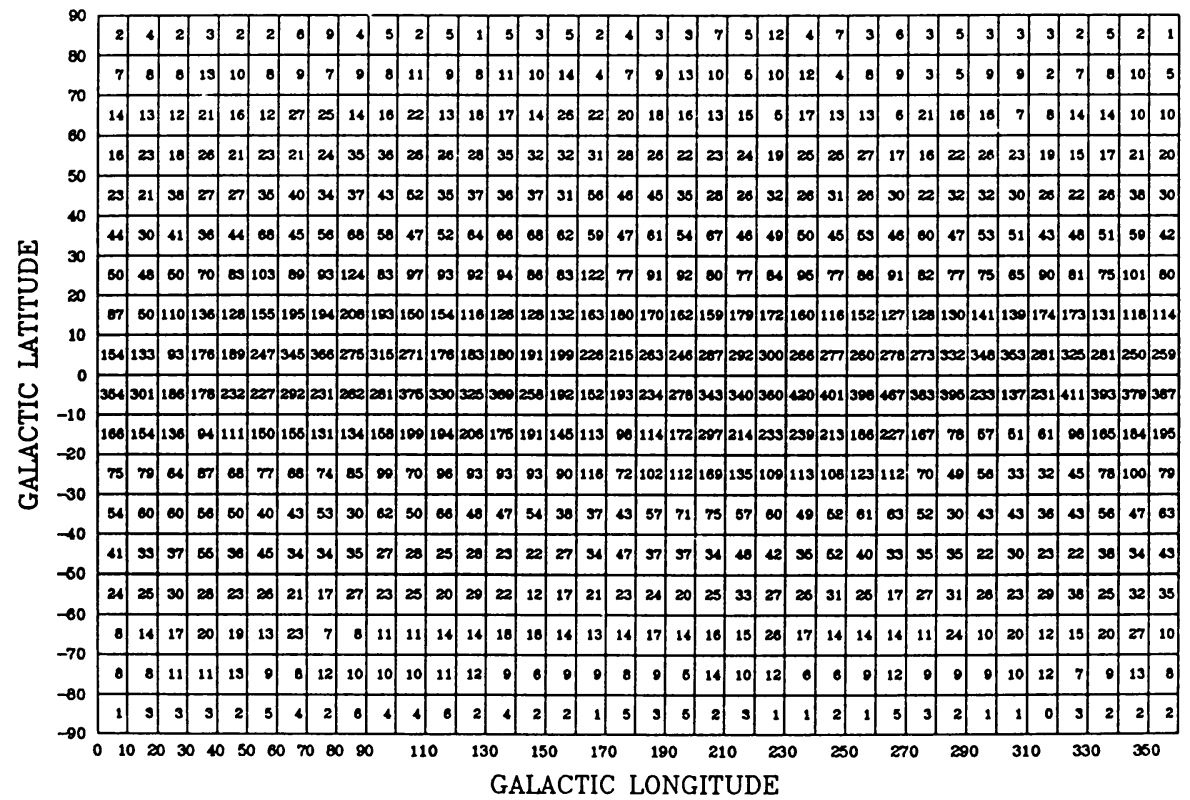

Figure 1. (upper panel) The distribution of stars in galactic coordinates. The $l=0^{\circ}$ is in the middle, and the galactic longitude increases towards the left. (lower panel) The number of stars in $10^{\circ} \times 10^{\circ}$ boxes. (RAL photo $89 \mathrm{MB} 3862$ ) 

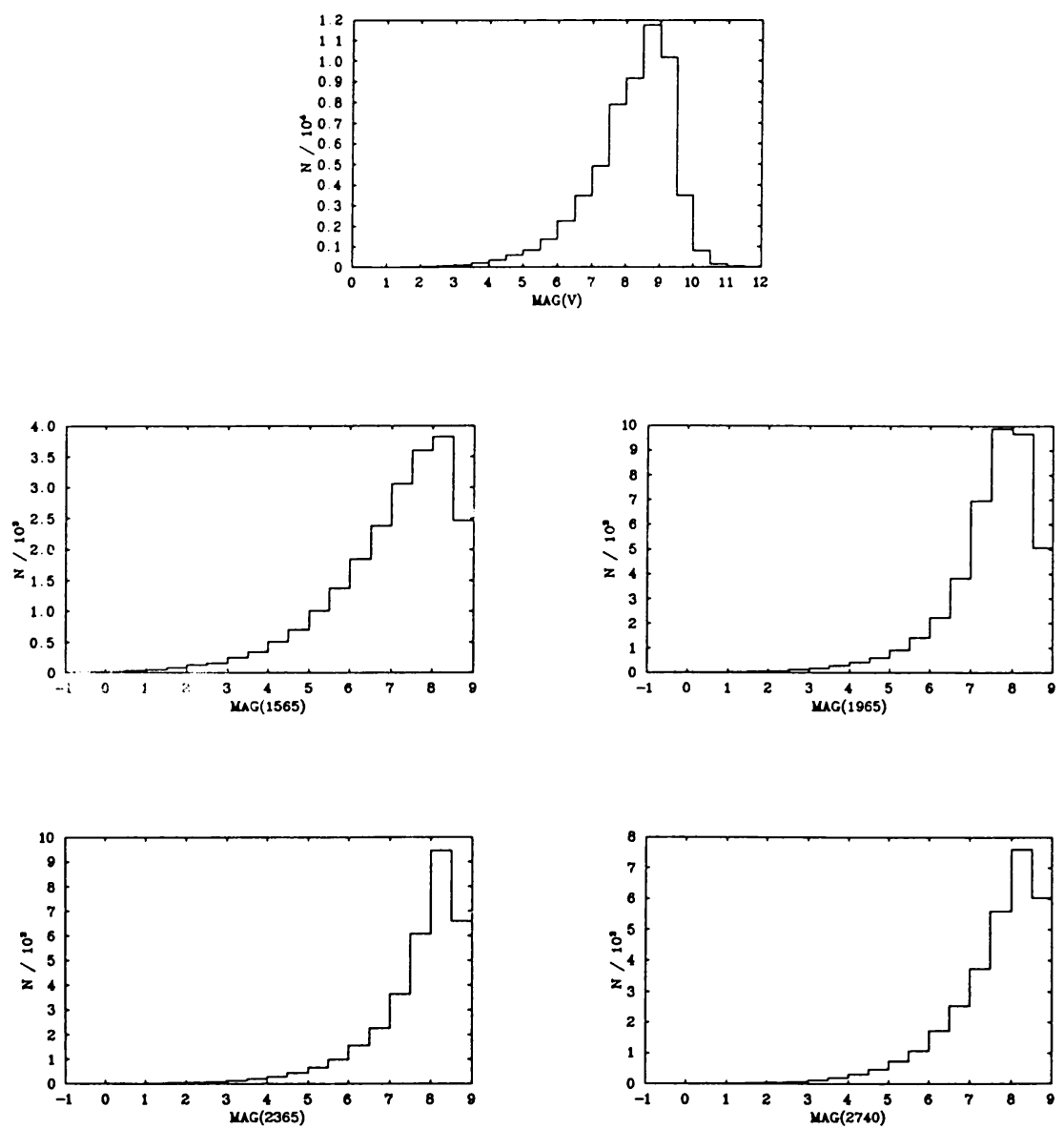

Figure 2. Distribution of visible and UV magnitudes of stars. The UV magnitudes have been obtained from observed fluxes. The cutoff at $m_{\mathrm{uv}} \approx 9$ is due to the lower flux limit imposed in selecting these stars. (RAL photo 89MB3960)

over $10^{\circ} \times 10^{\circ}$ boxes is shown in Figure 4. The distribution of stellar UV radiation in the Galaxy is asymmetric: there is more UV radiation in the southern hemisphere of the Galaxy than in the northern. Gould's belt (Stothers and Frogel 1974) is now well defined. This differs from the distribution of stars in the Galaxy (Figure 1); the star number density in the northern and the southern hemispheres (and in the Gould's belt) appears to be very similar, but the stars in the southern hemisphere (and in the Gould's belt) are UV-bright. This asymmetry was first predicted by Henry (1977) and observed by Henry et al. (1977). There is also an east-west asymmetry in the distribution of stellar UV radiation. More radiation is observed between longitudes $180^{\circ}$ and $360^{\circ}$ than between longitudes $0^{\circ}$ and $180^{\circ}$. The large semicircular region centered on $l=300^{\circ}$ is no longer so obvious, and, although there are fewer stars in this region, they are bright in the UV. In the galactic plane the sky appears brighter in directions where the interstellar dust is lower $\left(l=310^{\circ}\right)$. The region of the sky between longitudes $10^{\circ}$ and $50^{\circ}$ has low UV brightness; this region corresponds to a region of heavy reddening 
(Nandy et al. 1978). There are several bright stars off the galactic plane, and observations of an experiment with a large field of view (to detect diffuse UV radiation in the Galaxy) will require corrections for strong direct stellar radiation. The situation does not get any better with a smaller field of view as the radiation from faint stars (not shown here) will be comparable with the diffuse background radiation.
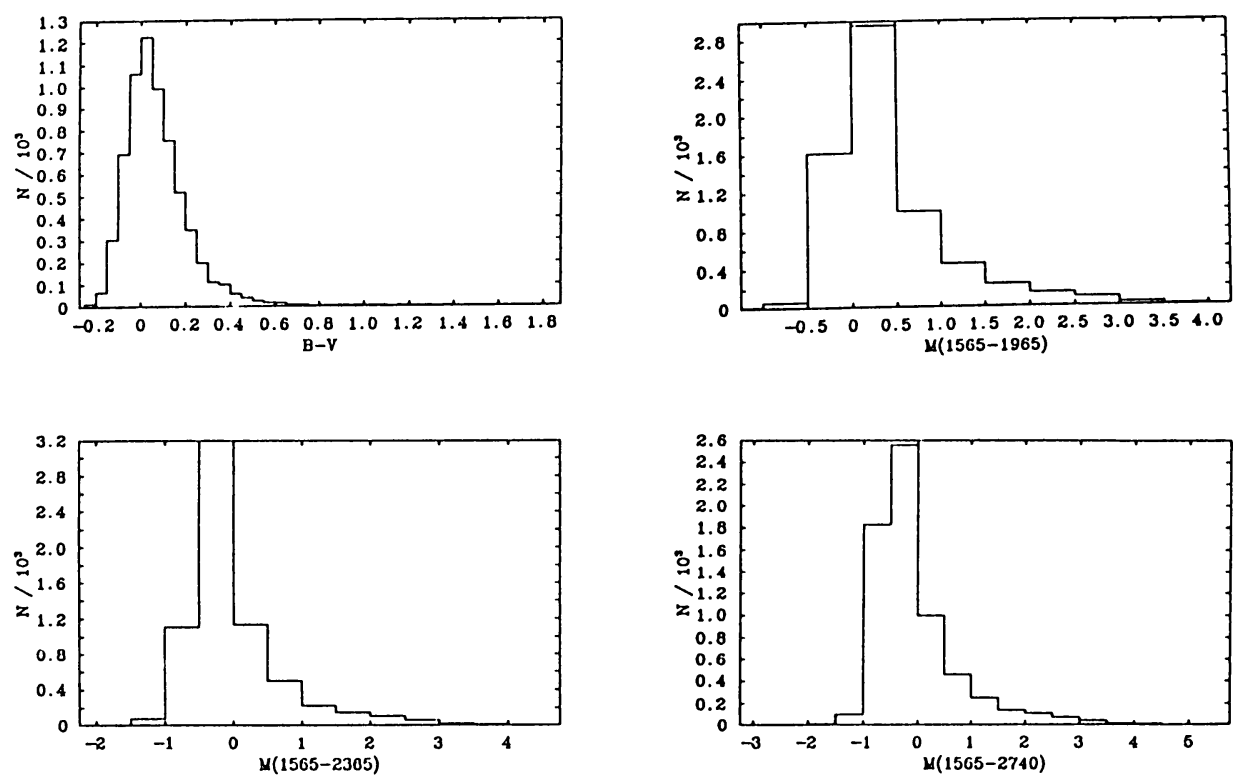

Figure 3. The distribution of visible and UV colors of stars. (RAL photo 89MB3959)

\subsection{Integrated Stellar Radiation}

The integrated stellar UV radiation is given in Table 3. Most of the radiation is emitted by B type stars. If a further division is made by subtypes (Gondhalekar, Phillips, and Wilson 1980), then it has been shown that most of the UV radiation is emitted by stars classified as B0 to B5. However, the integrated UV color of the Galaxy is that of an A0 star (Section 3), and this suggests a global reddening in the Galaxy. The value of the global color excess cannot be determined exactly because the stellar radiation is emitted by stars of different subtypes. But if it is assumed that the dominant subtype is B5 (and the color of the Galaxy is A0), then the global color excess $E_{(B-V)} \approx 0.27$. If, on the other hand, it is assumed that the dominant subtype is B3, then the global $E_{(B-V)} \approx 0.48$. The lower value of the global color excess is similar to the reddening per $\mathrm{kpc}$ in the plane of the Galaxy (Nandy et al. 1978). The higher value of the color excess would then suggest reddening in the galactic halo. However, the higher value of the global color excess is similar to the reddening in the plane of the Galaxy determined by Pandey and Mahra (1987)! The only possible conclusion at present is that the global color excess in the Galaxy is between 0.27 and 0.48 and that there may be some reddening off the plane of the Galaxy. 

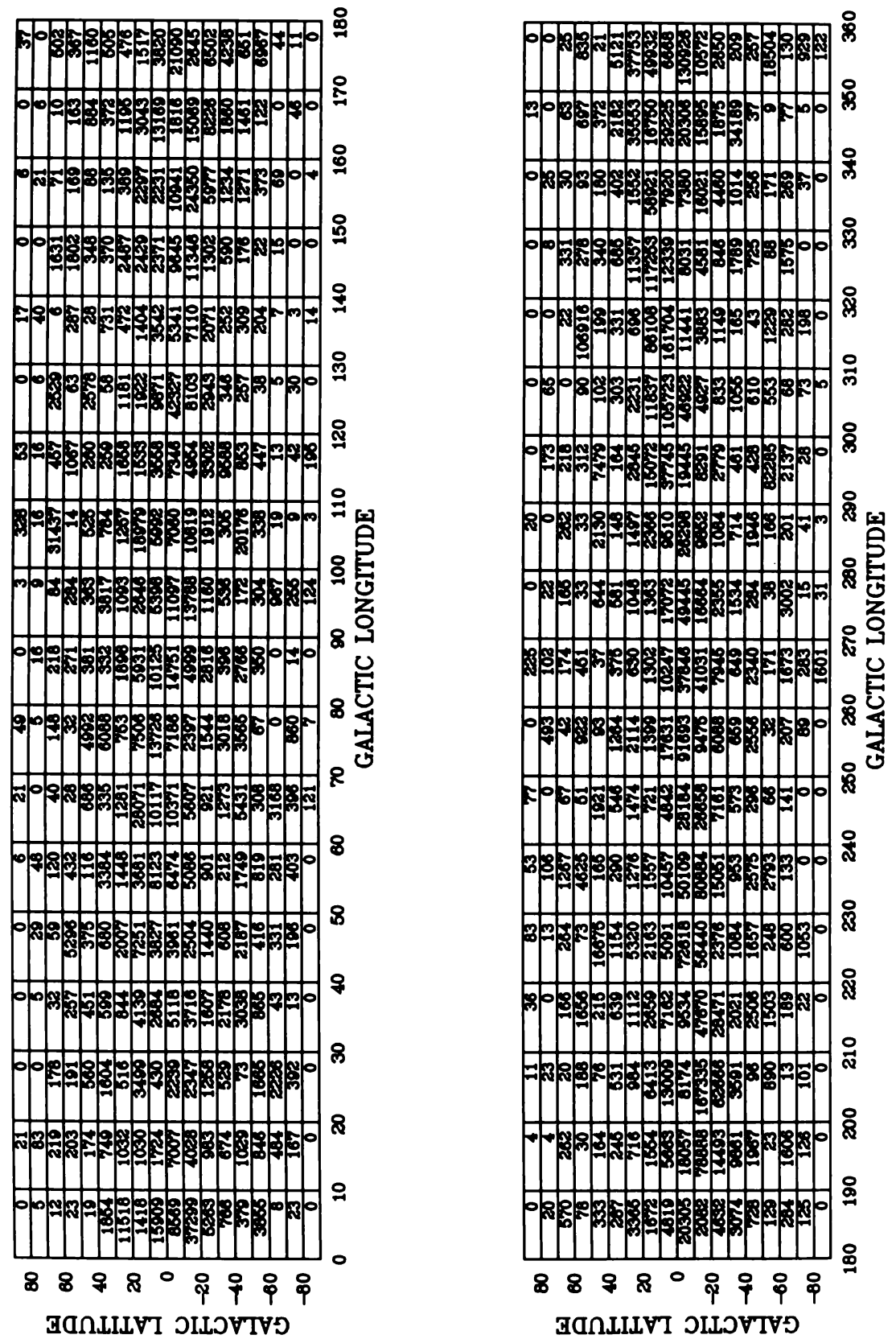

Figure 4a. The intensity of UV radiation at $1565 \AA$ (in $10^{-11} \mathrm{ergs} \mathrm{cm}^{-2} \mathrm{~s}^{-1} \AA^{-1} \mathrm{sr}^{-1}$ ) in $10^{\circ} \times 10^{\circ}$ boxes. (RAL photo $89 \mathrm{MB} 6526$ ) 

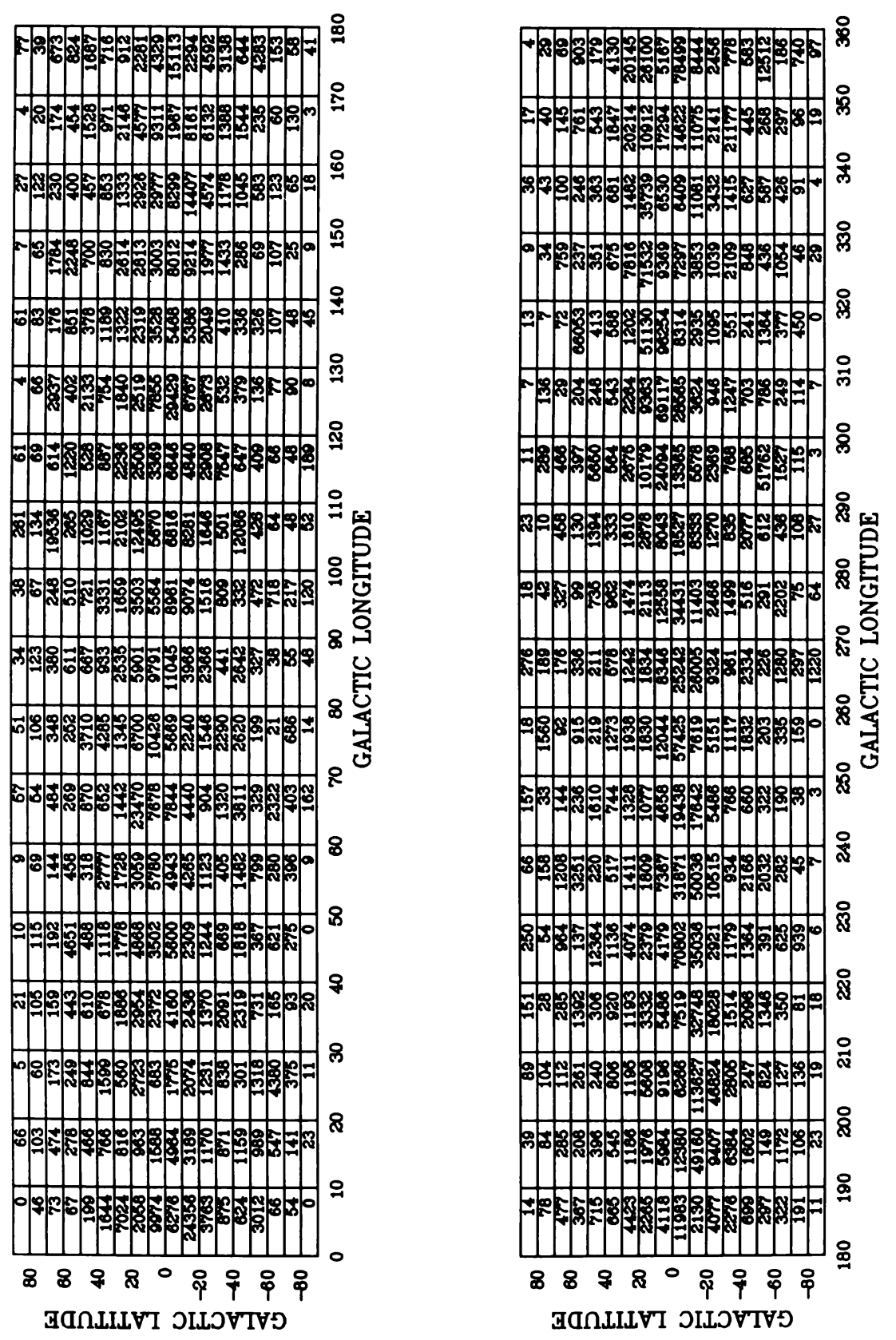

Figure 4b. The intensity of UV radiation at $1965 \AA$ (in $10^{-11} \mathrm{ergs} \mathrm{cm}^{-2} \mathrm{~s}^{-1} \AA^{-1} \mathrm{sr}^{-1}$ ) in $10^{\circ} \times 10^{\circ}$ boxes. (RAL photo 89MB6527) 

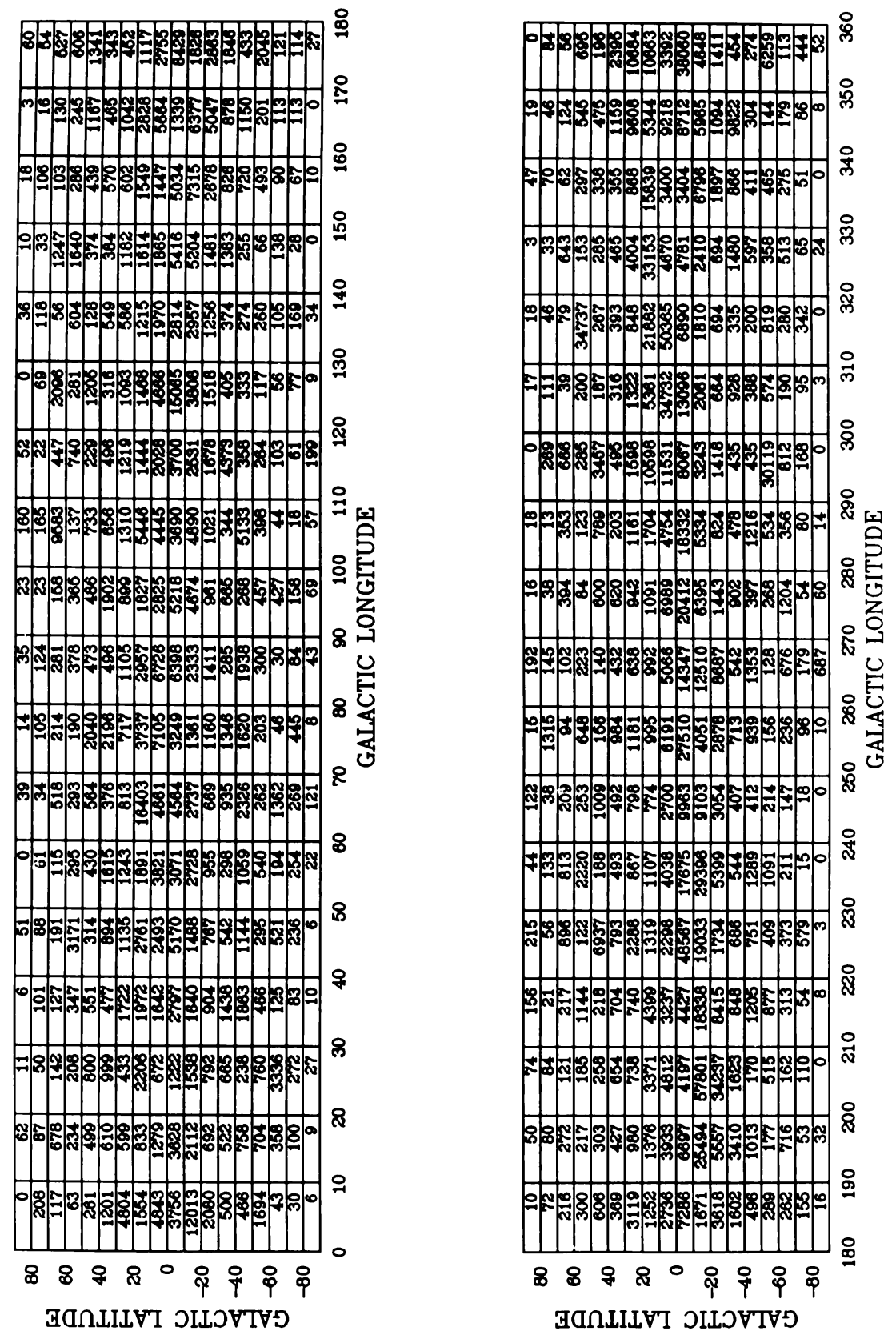

Figure 4c. The intensity of UV radiation at $2365 \AA$ (in $10^{-11} \mathrm{ergs} \mathrm{cm}^{-2} \mathrm{~s}^{-1} \AA^{-1} \mathrm{sr}^{-1}$ ) in $10^{\circ} \times 10^{\circ}$ boxes. (RAL photo 89MB6525) 

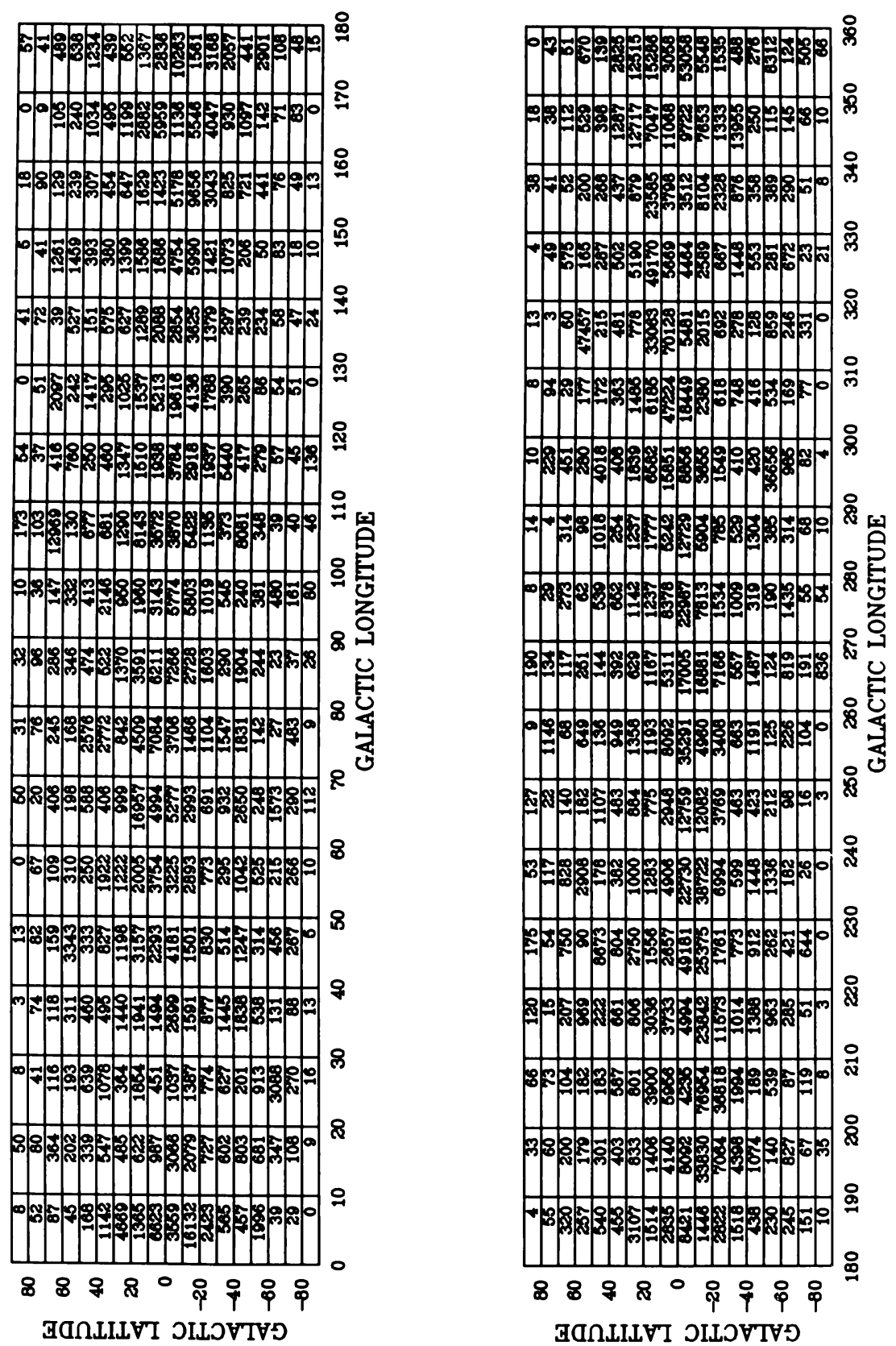

Figure 4d. The intensity of UV radiation at $2740 \AA$ (in $10^{-11} \mathrm{ergs} \mathrm{cm}^{-2} \mathrm{~s}^{-1} \AA^{-1} \mathrm{sr}^{-1}$ ) in $10^{\circ} \times 10^{\circ}$ boxes. (RAL photo 89MB6524) 
TABLE 3. Integrated Stellar Radiation in the Galaxy

(all longitudes, unit ergs $\mathrm{cm}^{-2} \mathrm{~s}^{-1} \AA^{-1}$ ) latitude $-90^{\circ}$ to $+90^{\circ}$

\begin{tabular}{lcccc}
\hline \hline spectral type & $1565 \AA$ & $1965 \AA$ & $2356 \AA$ & $2740 \AA$ \\
\hline $\mathrm{O}$ & $4.34(4)$ & $2.63(3)$ & $1.59(3)$ & $1.78(4)$ \\
$\mathrm{B}$ & $90.67(82)$ & $57.19(72)$ & $39.06(73)$ & $31.08(68)$ \\
$\mathrm{A}$ & $10.84(10)$ & $15.52(19)$ & $9.96(17)$ & $9.51(21)$ \\
$\mathrm{F}$ & 0.16 & 1.61 & 1.22 & $2.03(4)$ \\
$\mathrm{G}$ & 0.03 & 0.13 & 0.85 & 0.32 \\
others & $3.51(3)$ & $2.22(3)$ & $1.56(3)$ & $1.27(3)$ \\
Total & 109.52 & 79.31 & 53.48 & 45.39 \\
& \multicolumn{4}{c}{ Blue star radiation $\left(m_{1565}-2740 \leq 1.30\right)$} \\
& $40.90(37)$ & $23.70(30)$ & $15.50(29)$ & $10.61(23)$ \\
& Galactic plane radiation $\left(-45^{\circ} \leq b \leq+45^{\circ}\right)$ \\
& $97.45(89)$ & $69.89(88)$ & $46.91(88)$ & $39.67(87)$ \\
\hline
\end{tabular}

NOTE.-The figures in brackets are percentages of total radiation.

Carnochan and Wilson (1983) discovered a large population of stars more UV than are B8 stars, i.e., $m_{1565-2740} \leq-1.30$. A majority of these stars do not have published UBV colors or MK spectral types. The HD spectral types of these stars are from $\mathrm{O}$ to A. The globally integrated radiation emitted by these blue stars is given in Table 3; this radiation is $30 \%$ of the total integrated stellar radiation. A number of these stars are of MK spectral type B, but a large number are unclassified. Carnochan and Wilson have shown that the unclassified blue stars are unreddened subdwarfs and that the scale height of the hottest stars is similar to the scale height of the central stars of planetary nebulae. If there is a large population of these blue subdwarfs off the plane of the Galaxy, then they could be major contributors of UV radiation at mid and high latitudes. The implications, for the physics of the interstellar gas, of stellar UV radiation at high galactic latitudes have been investigated by Hills (1972), Terzian (1974), and Panagia and Terzian (1984).

\subsection{Galactic Model}

The spectrum of the integrated UV stellar radiation, obtained from the S2/68 observations, is shown in Figure 5. Also shown in this figure are various observations and modeled estimates of the direct UV starlight in the Galaxy. Apart from the summations of Henry et al. (1977) of UV radiation expected from stars listed in the Bright Star Catalogue and the SAO catalogue, most observations and models obtain integrated stellar radiation higher than the S2/68 observations. However, the integrated flux obtained from the S2/68 observations is a lower limit because of the flux limit imposed during selection of stars. The distribution of stellar flux per unit magnitude as a function of UV magnitude for $S 2 / 68$ observations has been given by Gondhalekar, Phillips, and Wilson (1980). There would be a cutoff at $m_{2740} \approx 9$ in this distribution for stars considered here. Incompleteness may not be a problem at $1565 \AA$, but the flux at $1965 \AA, 2365 \AA$, and $2740 \AA$ may have been underestimated. If it is assumed that the flux per unit magnitude drops linearly with magnitude, then the integrated flux at $1965 \AA$ should be revised up by $\sim 7 \%$, that at $2365 \AA$ by $\sim 10 \%$, and that at $2740 \AA$ by $\sim 20 \%$. Only the correction for the flux at $2740 \AA$ is perhaps significant. The integrated stellar radiation would still be underestimated as the $\mathbf{S} 2 / 68$ catalogue does not include stars whose fluxes are blended (because 
of the large entrance apertures of S2/68 photometer and spectrographs). Schemes for estimating the integrated flux from blended stars have been described by Gondhalekar, Phillips, and Wilson (1980) and Henry et al. (1988). Gondhalekar, Phillips, and Wilson have shown that the integrated stellar radiation is underestimated by about $12 \%$ if the blended stars are not included.

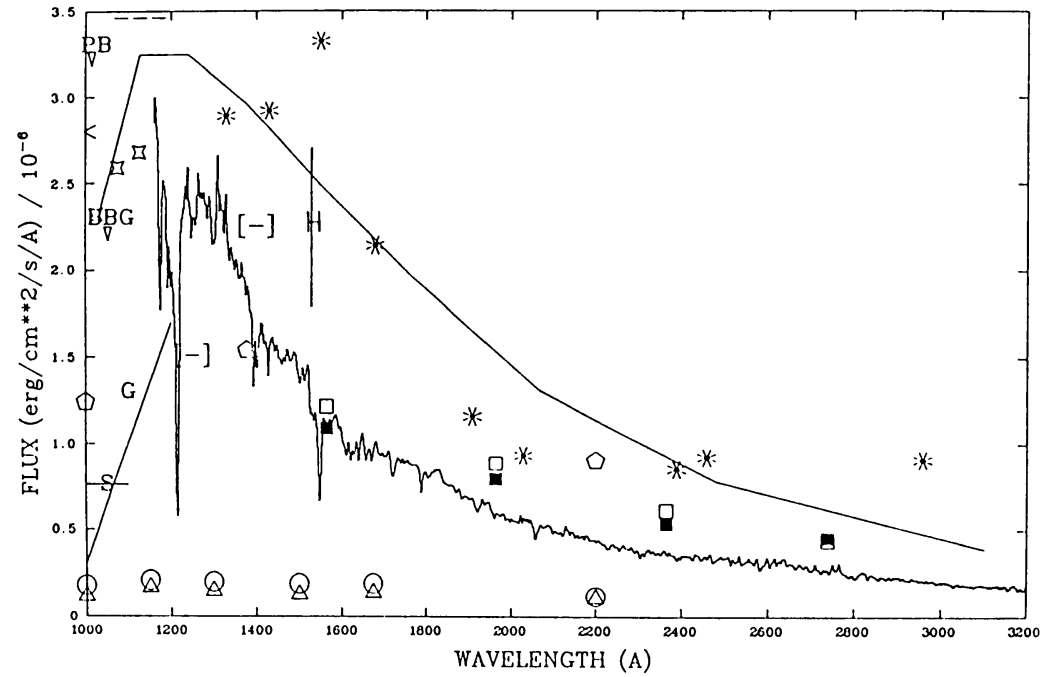

Figure 5. The spectrum of integrated direct UV radiation in the Galaxy. The integrated S2/68 broadband fluxes (filled squares), galactic model from IUE observations of unreddened stars (histogram), Gondhalekar, Phillips, and Wilson 1980 (open squares), Draine 1978 (connected straight lines), Jura 1974 (star), Witt and Johnson 1973 (asterisk), Habing 1968 (pentagon), Henry (BSC) 1977 (triangle), Henry (SAO) 1977 (circles), Paresce and Bowyer 1976 (PB), Belyaev et al. 1971 (---), Bixler, Bowyer, and Grewing 1984 (BBG), Grewing 1975 (straight line with $a$ G), Sandel, Shemansky, and Broadfoot 1979 (straight line with an S), Hayakawa, Yamashita, and Yoshioka 1969 ([-]), Henry et al. 1977 (vertical line with an $H$ ).

The integrated stellar UV radiation in the Galaxy can also be estimated from the stellar population of the Galaxy. Only the "parallel slab" model, in which the Galaxy is assumed to be a plane parallel slab (of infinite extent) with the Sun in the mid-plane, has been investigated in any detail (Gondhalekar and Wilson 1975). The integrated UV radiation can be obtained with the usual parameterization of stellar luminosity, the distribution of stars and dust off the galactic plane (Allen 1976), and the spectra of unreddened stars observed with IUE. The details have been given by Gondhalekar and Wilson and will not be repeated here. The modeled spectrum of the integrated starlight is shown in Figure 5. An average color excess $E_{B}-V=0.26 \mathrm{mag} \mathrm{kpc}^{-1}$ (Nandy et al. 1978) and the interstellar extinction curve of Seaton (1979) were used in this model. There is an embarrassingly good agreement between the modeled and the observed spectrum of the integrated radiation. But this agreement is deceptive; the latitude distribution of the observed and modeled starlight is shown in Figure 6, and there is total disagreement between the two distributions. The peak of the observed starlight is at $b \approx-5^{\circ}$ (due to Gould's belt) and that of the modeled starlight is at $b=0^{\circ}$. Also, the drop with latitude of the observed starlight is considerably faster than the drop of the modeled starlight. Most of the observed starlight is emitted between $-45^{\circ} \leq b \leq+45^{\circ}$ (Table 3 ), but the 
modeled starlight has almost equal contribution from all latitudes. This discrepancy is entirely due to the unrealistic parameterization of distribution of stars with the Galactic latitude.

The moral of the story is . . . use observed data whenever possible.
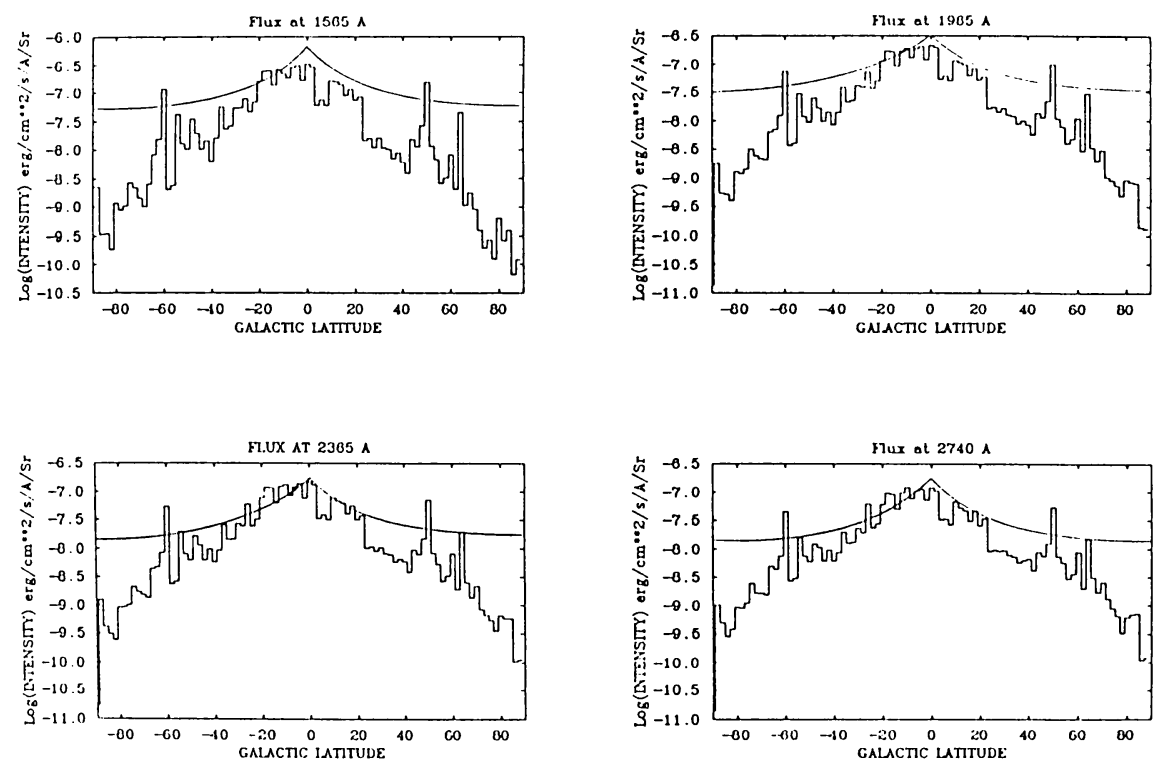

Figure 6. Comparison of the observed and modeled galactic latitude dependence of the intensity of direct stellar radiation.

\section{CONCLUSIONS}

1. The intensity of stellar UV radiation in the plane of the Galaxy and at mid-latitudes is probably known to an accuracy of $10-20 \%$.

2. The intensity of starlight at higher latitudes is less certain, especially if there is a large population of hot subdwarfs at higher latitudes.

3. Deep all-sky surveys in the UV are required to detect faint EUV stars. The ROSAT-WFC and $E U V E$ surveys will not be deep enough because of enhanced neutral hydrogen absorption in the EUV where these surveys will be made.

4. More realistic models of the stellar population in the Galaxy are required, particularly at high latitudes.

Acknowledgments - I a tape with the unpublished S2/68 catalogue and software for extracting data from this tape. P. T. Wallace and D. L. Giaretta are also thanked for providing invaluable assistance and various computer programs.

\section{REFERENCES}

Allen, C. W. 1976, Astrophysical Quantities (London: Athlone Press).

Bixler, J., Bowyer, S., and Grewing, M. 1984, Astron. Astrophys., 141, 422. 
Belyaev, V. P., et al. 1971, Cosmic Res., 8, 677.

Boksenberg, A., et al. 1973, MNRAS, 163, 291.

Bowyer, S. 1989, in Proc. IAU 139, Galactic and Extragalactic Background Radiation, ed. S. Bowyer and Ch. Leinert (Dordrecht: Kluwer Academic Publisher).

Carnochan, D. J. 1982, MNRAS, 201, 1139.

Carnochan. D. J., and Wilson, R. 1983, MNRAS, 202, 317.

Carruthers, G. R., and Page, T. 1984, Publ. Astron. Soc. Pacific, 96, 447.

Draine, B. T. 1978, Ap. J. Suppl., 36, 595.

Gondhalekar, P. M., and Wilson, R. 1975, Astron. Astrophys., 38, 329.

Gondhalekar, P. M., Phillips, A. P., and Wilson, R. 1980, Astron. Astrophys., 85, 272.

Habing, H. J. 1968, Bull. Astron. Inst. Neth., 19, 421.

Hayakawa, S., Yamashita, K., and Yoshioka, S. 1969, Astrophys. Space Sci., 5, 493.

Hayes, D. S., and Lathem, D. W. 1975, Ap. J., 197, 593.

Henry, R. C. 1977, Ap. J. Suppl., 33, 451.

Henry, R. C., et al. 1977, Ap. J., 212, 707.

Henry, R. C., et al. 1988, Atlas of the Ultraviolet Sky (Baltimore: The John Hopkins University Press).

Hills, J. G. 1972, Astron. Astrophys., 17, 155.

Jamar, C., et al. 1976, Ultraviolet Bright Star Spectrophotometric Catalogue, ESA SR-27.

Jura, M. 1974, Ap. J., 191, 375.

Kurt, V. G., and Sunyaev, R. A. 1968, Soviet Astron., 11, 928.

Lequeux, J. 1989, in Proc. IAU 139, Galactic and Extragalactic Background Radiation, ed. S. Bowyer and Ch. Leinert (Dordrecht: Kluwer Academic Publisher).

Macau-Hercot, D., et al. 1978, Supplement to the Ultraviolet Bright Star Spectrophotometric Catalogue, ESA SR-28.

Nandy, K., et al. 1978, MNRAS, 184, 733.

Onaka, T., et al. 1989, Ap. J., 342, 238.

Page, T., Carruthers, G. R., and Heckathorn, H. M. 1982, Revised S201 Catalogue of Far Ultraviolet Objects, Naval Research Laboratory Report 8487.

Pandey, A. K., and Mahra, H. S. 1987, MNRAS, 226, 635.

Paresce, F., and Bowyer, S. 1976, Ap. J., 207, 432.

Paresce, F. 1989, in Proc. IAU 139, Galactic and Extragalactic Background Radiation, ed. S. Bowyer and Ch. Leinert (Dordrecht: Kluwer Academic Publisher).

Sandel, B., Shemansky, D., and Broadfoot, A. 1979, Ap. J., 227, 808.

Seaton, M. J. 1979, MNRAS, 187, 73P.

Stothers, R., and Frogel, J. A. 1974, Astron. J., 79, 456.

Thompson, G. I., et al. 1978, Catalogue of Stellar Ultraviolet Fluxes, SRC.

Witt, A. N., and Johnson, M. W. 1973, Ap. J. 181, 363.

Witt, A. N. 1989, in Proc. IAU 139, Galactic and Extragalactic Background Radiation, ed. S. Bowyer and Ch. Leinert (Dordrecht: Kluwer Academic Publisher).

F. Paresce: What connection, if any, has been made between Carnochan and Wilson's population of excess blue stars and Green's recent survey of blue stars?

P.M. Gondhalekar: To my knowledge nobody has attempted to correlate the stars in Green's survey with UV blue stars discovered by Carnochan and Wilson. But it would be very interesting to do this correlation as Green's survey goes deeper than the observations of Carnochan and Wilson and it should be possible to identify blue objects off the Galactic plane.

G. Szencsenyi-Nagy: Your results demonstrated that the integrated light (and colours) of the galaxy can be best described as the light of $A$ stars. The spectra of these objects are dominated by very strong Balmer lines. Do you think that the integrated spectrum of the Galaxy is also dominated by these absorption features?

P.M. Gondhalekar: The UV colours of the Galaxy are similar to the colours of A stars, but this is due to reddening of early B type stars which are the major emitters of UV radiation. For a synthetic spectrum of the Milky Way see Mattila's review (this volume). 